lange Zeit zum Standardwerk zur Geschichte der sowjetischen Lager emporhob, die der Meinung war, dass dies das Buch sei, „auf das Solženizyn gehofft" (Wall Street Journal) habe, zeigt leider, dass es hier nicht um den Stellenwert von Wissenschaft und Forschung geht, sondern um politisches agenda-setting. So lobenswert es sein mag, die politisch wichtigen, richtigen und zum Teil leider arg vernachlässigten Themen einem breiten Publikum zu Bewusstsein zu bringen, so sehr schadet mangelnde Seriosität auf längere Sicht. Diese mangelnde Qualität hat möglicherweise auch damit zu tun, was in Deutschland mit der rigorosen Spar- und Abwicklungspolitik osteuropabezogener Forschung und Lehre leider schon seit vielen Jahren schlechte Wirklichkeit ist, und sich auch in den USA immer stärker bemerkbar macht. Mit dem Argument, dass der Kalte Krieg vorbei und das sowjetische Imperium untergegangen sei, werden auch jenseits des Atlantiks die Mittel für osteuropabezogene Forschung und Lehre immer stärker gekürzt (vgl. dazu: Stephen E. Hanson/Blair A. Ruble, Rebuilding Russian Studies; in: Problems of Post-Communism, Vol. 52 (2005), Nr. 3, S. 49-57). Insofern bleibt zu hoffen, dass die breite öffentliche Rezeption, die Applebaums Buch erfahren hat, eine diesbezügliche Trendwende einleitet.

\section{INTERNATIONALE POLITIK}

\author{
Andreas Rechkemmer: Postmodern Global Go- \\ vernance. The United Nations Convention \\ to Combat Desertification. Baden-Baden: \\ Nomos 2004, 172 S., € 29,--
}

\section{Helmut Breitmeier}

Im Jahr 1994 wurde als Folge des Rio-Prozesses die UN-Konvention zur Bekämpfung der Wüstenbildung verabschiedet. In der vorliegenden Studie wird diese Konvention als ein signifikantes Beispiel für die Herausbildung postmoderner Formen des Regierens jenseits des $\mathrm{Na}$ tionalstaates angesehen. Die Arbeit beinhaltet im strengen Sinne gesehen keine Hypothesen.
Vielmehr wird versucht, spezifische Eigenschaften des Regimes zur Bekämpfung der Wüstenbildung zu identifizieren, welche dieses als eine postmoderne Institution ausweisen. Auf der Grundlage einer Sichtung verschiedener postmoderner Ansätze kommt der Autor im theoretischen Teil der Arbeit zu dem Fazit, dass sich in der Weltpolitik ein post-westfälisches Paradigma entwickelt. Dieses beinhaltet den Wandel der Kontexte und Kerninstitutionen bzw. Normen und die Relativierung der Bedeutung von Akteuren (z.B. den Nationalstaat), die für das Westfälische Staatensystem grundlegend waren. Im Rio-Prozess, der in der UN-Konferenz über Umwelt und Entwicklung gipfelte und in der Folgezeit mehrere Überprüfungskonferenzen nach sich zog, sieht der Autor eine Reihe von Phänomenen zum Ausdruck kommen, welche die Herausbildung einer postwestfälischen Weltordnung veranschaulichen: z.B. die Errichtung globaler Regelwerke zur Bearbeitung grenzüberschreitender Problemlagen, die Rolle des Wissens für die globale Problemlösung oder den Mix unterschiedlicher Formen des Regierens. In zwei etwas umfangreicheren Kapiteln wird der Prozess der Entstehung und Weiterentwicklung des Regimes beschrieben. In diesen Kapiteln wird herausgearbeitet, inwiefern sich die Tendenz zur Herausbildung einer post-westfälischen Ordnung in der Wüstenbildungskonvention niederschlägt. Die Konvention wird als ein Kontext dargestellt, in dem komplexe bzw. problemfeldübergreifende Politiken auf unterschiedlichen Ebenen zur Anwendung kommen, in dem Politiknetzwerke und Nichtregierungsakteure in Prozesse der Politikformulierung und -implementation einbezogen werden und in dem neue Instrumente zur Effektivitätssteigerung des Regierens eingesetzt werden. Der in der Konvention zur Bekämpfung der Wüstenbildung enthaltene „bottom-up-approach" wird beispielsweise besonders hervorgehoben und als "one of the most radical conceptual results of post-Westphalian politics" (90) bezeichnet. Der etwas kurz gehaltene empirische Teil der Studie vermittelt nützliche Einblicke in die praktische Arbeit des Regimes zur Bekämpfung der Wüstenbildung. Offen bleibt indessen, wie stark dieses Regime mittel- und langfristig zur Eindämmung dieses grundlegenden Problems beitragen kann. 